\title{
Serum concentration of immunoglobulin G-type antibodies against the whole Epstein-Barr nuclear antigen 1 and its aa35-58 or aa398-404 fragments in the sera of patients with systemic lupus erythematosus and multiple sclerosis
}

D. Csuka, ${ }^{\star}$ D. Simon, ${ }^{\dagger}$ R. Hóbor, ${ }^{\ddagger}$

K. Uray ${ }^{\S}$ Z. Prohászka, ${ }^{\star}$ Z. Bánlaki, ${ }^{\star}$

P. K. Jani, ${ }^{\star}$ Á. Szilágyi, ${ }^{\star}$ F. Hudecz, ${ }^{\S}$

K. Rajczy, ${ }^{9}$ G. Beke, ${ }^{\ddagger}$ A. Boros Major,

A. Tordai, ${ }^{9}$ Z. Illés, ${ }^{\star *}$ T. Berki, ${ }^{\dagger}$

L. Czirják ${ }^{\ddagger}$ and G. Füst*

${ }^{*}$ Third Department of Internal Medicine, Semmelweis University, ${ }^{5}$ Research Group of Peptide Chemistry, Hungarian Academy of Sciences, Eötvös L. University, 'Hungarian National Blood Transfusion Service, Budapest, ${ }^{\dagger}$ Department of Immunology and Biotechnology, ${ }^{\ddagger}$ Department of Rheumatology and Immunology, Medical Center, and ${ }^{* *}$ Division of Clinical and Experimental Neuroimmunology, University of Pecs, Pecs, Hungary

Accepted for publication 29 October 2012 Correspondence: D. Csuka, Third Department of Internal Medicine, Semmelweis University, Budapest, Kútvölgyi Str 4, H-1125 Budapest, Hungary.

E-mail: csukadorka@gmail.com

\begin{abstract}
Summary
Several studies suggest that infection by Epstein-Barr virus (EBV) might be one of the environmental factors which facilitates the development of autoimmune disorders in genetically susceptible individuals. Recent data indicate that high anti-Epstein-Barr nuclear antigen 1 (EBNA)-1 immunoglobulin (Ig)G titre is a strong risk factor for multiple sclerosis (MS) in patients both with and without the main genetic predisposing trait, human leucocyte antigen (HLA)-DRB1 ${ }^{\star}$ 15:01. Because no similar studies have been published in systemic lupus erythematosus (SLE) patients, we determined the HLA-DRB1 ${ }^{\star}$ 15:01 carrier state and the serum titres against the whole EBNA-1 and its small fragments aa35-58 and aa398-404 in 301 SLE patients, 135 MS patients and in 345 healthy controls. The carrier state of the HLADRB1 ${ }^{\star} 15: 01$ allele was deduced from genotyping of a tagSNP ( $r$ s3135388) by applying a Taqman-based assay. The serum concentrations of antibodies to EBNA-1 and its aa35-58 or aa398-404 fragments were determined using a commercial assay (ETI-EBNA-G) and home-made enzyme-linked immunosorbent assays, respectively. The serum concentration of anti-EBNA-1 antibodies was significantly $(P<0.001)$ higher both in MS and SLE patients than in controls. Similar significant differences were found both in HLADRB1 ${ }^{\star}$ 15:01 carriers and non-carriers. Furthermore, titres of antibodies against the aa35-58 EBNA-1 fragment were elevated both in MS and SLE patients. By contrast, the levels of aa398-404 EBNA-1 antibodies were elevated significantly only in the SLE patients. These findings indicate that high anti-EBNA-1 IgG titres are HLA-DRB1 ${ }^{\star} 15: 01$-independent risk factors not only for MS, but also for SLE, while high antibody titres against the aa398-404 fragment are characteristic for SLE.
\end{abstract}

Keywords: Epstein-Barr virus, HLA, multiple sclerosis, systemic lupus erythematosus

\section{Introduction}

Several studies indicate that infection by the Epstein-Barr virus (EBV) is associated strongly with certain autoimmune diseases, such as multiple sclerosis (MS), systemic lupus erythematosus (SLE), rheumatoid arthritis (RA) or myasthenia gravis (MG) [1]. Most studies addressed the EBV association of the former two [2-4]. Perhaps the strongest evidence for the association of the EBV infection and MS is the observation that patients with a high
Epstein-Barr nuclear antigen 1 (EBNA-1) immunoglobulin (Ig)G titre face a several-fold higher chance of developing MS subsequently, compared to those with a low titre. Some papers, published several years ago $[5,6]$, have already reported that the titres of certain antibodies against EBV (EBNA-1, EBNA-2 and EA-D) are higher in blood samples obtained from future patients before the onset of MS than in those from age- and sex-matched controls. Recently, four longitudinal studies [7-10] provided strong support for these findings. In 2005, Levin et al. [7] 
measured the titres of various antibodies against EBV in serial blood samples from 83 patients with MS and controls, using banked blood samples and archived data maintained by the US Army. Beyond the age of 20 years, anti-EBNA-1 titres were significantly higher in the samples from future patients, but remained unchanged in those from controls. Studies by Levin et al. showed that people who have not yet been infected by EBV face an extremely low chance of contracting MS; however, the risk increases dramatically after infection [9]. Theoretically, regarding the relationship between high anti-EBNA-1 antibody level and MS, it could be hypothesized that the susceptibility to MS and predisposition to the elevation of antibody titre might be attributed to the same genetic factors. However, this assumption has been largely debated by the latest studies [9,11-13] that compared the presence of human leucocyte antigen (HLA)$\mathrm{DRB}^{*}{ }^{*}$ 15:01 (the strongest hereditary risk factor for MS) and high titres of anti-EBNA-1 IgG antibodies in patients with MS and in healthy controls. As demonstrated by these studies, both indicate a high risk for MS but are independent of each other. The evidence for the association between high titres of the anti-EBV antibodies and SLE is much more limited than that obtained in MS patients $[14,15]$. In a recent new study, Esen et al. [16] did not find any difference between the anti-EBNA-1 antibody levels of SLE patients and controls; however, in agreement with earlier findings [17], they detected significantly higher levels of antibodies reacting with the EA/D antigen in the patients. The HLA$\mathrm{DRB}^{*} 15: 01$ allele is less important in the genetic background of SLE than that of MS; however, several studies also indicate an elevated risk of SLE in the carriers of this allele or haplotype [18-20].

No studies have yet been performed to clarify whether the genetic factors and the high titres of anti-EBV antibodies are independent and additive risk factors not only for MS, but also for SLE. Therefore, the primary aim of the present work was to compare the levels of anti-EBNA-1 antibodies in patients with MS or SLE and in healthy controls, as well as to determine simultaneously the proportion of the carriers of the HLA-DRB1 ${ }^{\star} 15: 01$ allele in these three groups. The secondary objective of the study was to compare the fine specificity of the anti-EBNA-1 antibodies in the two diseases investigated. Several studies in SLE patients and experimental animals demonstrated a strong cross-reaction between anti-Sm $\mathrm{B}^{\prime} / \mathrm{B}$ antibodies and those to an epitope (aa398-404, PPPGRRP) of the EBNA-1 molecule [21-24]. Furthermore, the N-terminal 35-58 sequence of EBNA-1 is highly homologous to the C-terminal 95-119 region of the ribonucleoprotein $\mathrm{SmD}$ [25]. The levels of the antibodies for the former epitope, however, have not been tested in the sera of MS patients. Therefore we introduced new enzyme-linked immunosorbent assay (ELISA) methods for measuring the serum concentrations of antibodies against the EBNA-1 fragments aa35-58 and aa398-404, and compared the median levels in the sera of high number of SLE and MS patients and healthy controls.

\section{Patients and methods}

\section{Subjects tested}

A total of 301 SLE patients [275 females, aged 44.0 (37.0$57 \cdot 0)$ years] were included into the study. The median [interquartile (IQ) range] the age of the patients at the onset of disease was $28 \cdot 0(21 \cdot 0-41 \cdot 0)$ years; $6 \cdot 0(3 \cdot 0-12 \cdot 0)$ years elapsed between diagnosis and blood sampling, whereas the patients were followed-up for $7 \cdot 0(2 \cdot 0-12 \cdot 0)$ years from the diagnosis of SLE. The SLE disease activity index (SLEDAI) score at the time of blood sampling was $4 \cdot 0$ $(2 \cdot 0-8 \cdot 0)$.

The most frequent organ manifestations included skin manifestations $(86 \%)$, polyarthritis $(71 \%)$, cytopenia (58\%), central nervous system (CNS) manifestations (33\%), nephritis (32\%), pulmonary symptoms (17\%), lymphadenopathy $(15 \%)$ and cardiac (12\%) manifestations, as well as myopathy (9\%) (percentages of the whole SLE patient group). In detail, SLE patients experienced the following symptoms: anaemia (49\%), leucopenia (44\%), alopecia $(16 \%)$, thrombopenia $(15 \%)$, pericarditis (11\%), mental dysfunction (7\%), serositis $(6 \%)$, vasculitis $(3 \%)$, erythema of palm/sole (3\%) and myocarditis (2\%).

Cyclophosphamide was administered to $8 \%$ of patients, and azathioprine was used in $28 \%$ of patients. Thirteen per cent of SLE patients received both these immunosuppressants, whereas $51 \%$ of the SLE patients received none of these drugs. Steroid preparations were used in $80 \%$ of the patients, either transiently or continuously.

One hundred and thirty-five MS patients [76 females, aged $41 \cdot 0(34 \cdot 8-48 \cdot 0)$ years] were included into the study. The median (IQ range) the age of the patients at the onset of the disease was $30 \cdot 0(25 \cdot 0-34 \cdot 5)$ years, and $5 \cdot 1(1 \cdot 0-9 \cdot 5)$ years elapsed between the onset of disease and blood sampling. The patients were followed for a mean $11.0(7 \cdot 0$ $17 \cdot 0)$ years from the diagnosis of MS. The mean Expanded Disability Status Scale (EDSS) scores for the cohort were 1.7 (range: 1.0-3.5) at the time of diagnosis of MS and 2.5 (range: $1 \cdot 0-4 \cdot 0$ ) at the time of blood sampling. Seventy-five patients had oligoclonal bands (OCB) in the cerebrospinal fluid. The distribution of the subtypes of MS were as follows (percentage of the whole MS patient group): 73\% with relapsing-remitting MS, $14 \%$ with secondary progressive MS, $8 \%$ with primary progressive MS and 5\% with clinically isolated syndrome (including relapsing inflammatory optic neuropathy). MS patients were treated as follows at the time of blood sampling: $11 \%$ with intramuscular (i.m.) interferon (IFN)- $\beta 1 \mathrm{a}, 10 \%$ with subcutaneous (s.c.) glatiramer acetate, $8 \%$ with s.c. IFN- $\beta 1$ a, $4 \%$ with s.c. IFN$\beta$ b, $2 \%$ with intravenous (i.v.) mitoxantrone and $1 \%$ with 
oral fingolimod. Sixty-four per cent of the patients received no treatment at the time of blood sampling.

Healthy controls, comprising 107 subjects who had volunteered at the time of a regular medical screening, as well as 238 parents of patients scheduled for bone marrow transplantation - that is, 345 individuals [ 189 females, aged $46 \cdot 0(37 \cdot 0-57 \cdot 0)$ years] in total - were included.

\section{Genetic determination}

The carrier state of the HLA-DRB1*15:01 allele was deduced from genotyping of a tagSNP (rs3135388), by applying a Taqman-based assay [26].

\section{Synthesis of the aa398-404 EBNA-1 peptide conjugate}

Because of the small size of the EBNA-1 epitope aa398404, it was not possible to use the peptide as a target antigen in ELISA; therefore, this peptide was prepared as a multiple antigenic peptide (MAP) conjugate to facilitate its adsorption to the ELISA plates. ( ${ }^{398}$ Pro-Pro-ProGly-Arg-Arg-Pro ${ }^{404}$-Ttds)-Lys $\left({ }^{398}\right.$ Pro-Pro-Pro-Gly-Arg-ArgPro $^{404}$-Ttds)-Lys [( ${ }^{398}$ Pro-Pro-Pro-Gly-Arg-Arg-Pro ${ }^{404}$-Ttds) -Lys $\left({ }^{398}\right.$ Pro-Pro-Pro-Gly-Arg-Arg-Pro ${ }^{404}$-Ttds) $]-\mathrm{NH}_{2}$ (aa398 -404 EBNA-1 peptide conjugate) was prepared by the solid-phase peptide synthesis method on 4methylbenzhydrylamine resin, with a mixed Boc/Bzl-Fmoc/ ${ }^{t} \mathrm{Bu}$ strategy. First, the Boc-Lys(Boc) amino acid derivative was coupled to the resin with diisopropylcarbodiimide/ 1-hidroxybenzotriazol coupling agents. Then, after cleaving the Boc protecting group with $33 \%(\mathrm{~V} / \mathrm{V})$ trifluoroacetic acid (TFA), dichloromethane, Boc-Lys(Boc) was coupled again and the protecting groups were removed, thus producing a tetramer dendrimer or multiple antigenic peptide (MAP) core structure [27]. To enhance the solubility of the conjugate, Fmoc-4,7,10-trioxa-1, 13-tridecanediaminosuccinic acid (Fmoc-Ttds) [28] was coupled to all four amino groups, and then the aa398-404 EBNA-1 peptide chains were built up with with Fmoc/ ${ }^{t} \mathrm{Bu}$ strategy. Once the synthesis was complete, the side chain protecting groups were cleaved with TFA, a mixture of 1,2-ethanedithiol, thioanisole, phenol and water $(10: 0 \cdot 125: 0 \cdot 25: 0 \cdot 75: 0 \cdot 25 \mathrm{~V} / \mathrm{V} / \mathrm{V} / \mathrm{m} / \mathrm{V})$ for $2 \cdot 5 \mathrm{~h}$. Then, the peptide conjugate was cleaved from the resin with liquid hydrogenfluoride. The peptide conjugate was purified by reverse-phase high-performance liquid chromatography (RP-HPLC); its homogeneity was determined by analytical RP-HPLC and was found to be $>90 \%$ pure. It was identified by a Bruker Esquire $3000+$ iontrap mass spectrometer ( $\mathrm{M}_{\text {calc: }}$ 4642·6, $\left.\mathrm{M}_{\text {meas }}: 4644 \cdot 4\right)$, and its amino acid composition was verified after acidic hydrolysis and post-column ninhydrin derivatization on a SYKAM automated amino acid analyser [Pro: $15 \cdot 5$ (16), Gly: 4.08 (4), Lys: $3 \cdot 3$ (3), Arg: $7 \cdot 04(8)]$.

\section{Synthesis of aa35-58 EBNA-1 peptide}

Peptide $\quad{ }^{35}$ GGDNHGRGRGRGRGRGGGRPGAPG ${ }^{58}-\mathrm{NH}_{2}$ (EBNA-1 aa35-58) was prepared by the solid-phase peptide synthesis method on 4-methylbenzhydrylamine resin, with Fmoc/t'Bu strategy. The Fmoc-amino acid residues were coupled with diisopropylcarbodiimide/ 1-hydroxybenzotriazol or O-benzotriazole-N,N, $\mathrm{N}^{\prime}, \mathrm{N}^{\prime}$ tetramethyl-uronium-hexafluoro-phosphate/N,N-diisopro pylethylamine/1-hidroxybenzotriazol coupling agents and the Fmoc group was cleaved with piperidine1,8-diazabicyclo[5·4.0] undec-7-ene-DMF (2:2:98 V/V/V). After the completion of the synthesis the side chain protecting groups and the peptide were cleaved with TFA: a mixture of 1,2-ethanedithiol, thioanisole, phenol and water

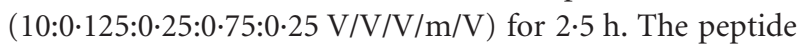
conjugate was purified by RP-HPLC; its homogeneity was determined by analytical RP-HPLC and was found to be $>90 \%$ pure. It was identified by a Bruker Esquire $3000+$ iontrap mass spectrometer $\left(\mathrm{M}_{\text {calc }}: 2270 \cdot 4, \mathrm{M}_{\text {meas }}\right.$ : 2271.9), and its amino acid composition was verified after acidic hydrolysis and post-column ninhydrin derivatization on a SYKAM automated amino acid analyser Asp/Asn: 1.59 (2) (hydrolytic loss), Pro: $2 \cdot 61$ (2), Gly+Ala: $12 \cdot 2(12+1)$ (determined as co-eluted hydrolysis products), His: 1.08 (1), Arg: 6.53 (6).

\section{Measurement of the serum concentration of the IgG-type antibodies for the whole EBNA-1 molecule and the aa35-58 or aa398-404 EBNA-1 peptide regions}

The serum concentration of antibodies to EBNA-1 was determined using a commercial enzyme-linked immunosorbent assay (ETI-EBNA-G; DiaSorin, Saluggia, Italy). The plates of the kit were coated with a 59-amino-acid synthetic peptide containing several domains of the EBNA-1, which are known as immunodominant epitopes of EBNA-1. The Gly-Ala repeat was excluded, owing to its known crossreactivity with autoantigens. Interassay coefficient of variation was found to be $5 \%$, while intra-assay coefficient of variation was $4 \%$.

The levels of antibodies to the aa35-58 or aa398-404 EBNA-1 epitope regions were measured by home-made ELISA methods, as follows. Maxisorb ELISA plates (Nunc ${ }^{\mathrm{TM}}$, Roskilde, Denmark) were coated with $10 \mu \mathrm{g} / \mathrm{ml}$ of the aa35-58 peptide or aa398-404 conjugate of EBNA-1 in phosphate-buffered saline (PBS), $100 \mu \mathrm{l} /$ well $\left(\mathrm{ON}, 4^{\circ} \mathrm{C}\right.$ ). After blocking the plates with blocking buffer [PBS, 3\% bovine serum albumin (BSA), $0 \cdot 05 \%$ Tween 20] for $1 \mathrm{~h}$ at room temperature (RT), the serum samples, as well as the positive and negative controls, were diluted 1:40 for the anti-aa35-58 EBNA-1 detection or 1:100 for the antiaa398-404 EBNA-1 detection in dilution buffer (PBS containing $0 \cdot 05 \%$ Tween 20 and 2\% BSA). A standard, twofold dilution series (1:10-1:640) of pooled serum was applied to 
each plate along with diluted analytes in duplicate, and incubated for $1 \mathrm{~h}$ at RT. The standard serum pool was prepared from 20 individual samples which contained high levels of antibodies against EBNA-1. Plates were then washed with PBS-T (PBS, 0.05\% Tween 20), and incubated with peroxidase-conjugated rabbit anti-human IgG (Jackson Immunoresearch, West Grove, PA, USA), 1:5000 diluted in dilution buffer ( $1 \mathrm{~h}, \mathrm{RT})$. After washing steps, $100 \mu \mathrm{l} /$ well $o$-phenylenediamine dihydrochloride (OPD) substrate (Dako, Glostrup, Denmark) was applied and the plates were developed for $15 \mathrm{~min}$ at RT. The enzymatic reaction was terminated with $50 \mu \mathrm{l} /$ well $1 \mathrm{M}$ sulphuric acid and optical density was measured at 492/620 nm. The concentrations are expressed in arbitrary units; 100 units correspond to the levels of anti-aa35-58 EBNA-1 antibody or anti-aa398-404 EBNA-1 antibody in $1 \mathrm{ml}$ undiluted standard serum pool. Interassay coefficient of variation was found to be $8 \%$, while intra-assay coefficient of variation was $6 \%$ for the anti-aa35-58 EBNA-1 assay; the corresponding values were $7 \%$ and $6 \%$ for the anti-aa398-404 EBNA-1 assay, respectively.

\section{Determination of anti-SmB/B' and anti-SmD titres}

Commercial kits were used for the determination of anti$\mathrm{SmB} / \mathrm{B}^{\prime}$ titres (Varelisa ${ }^{\circledR}$ Sm antibody assay; ThermoFisher Scientific, Phadia GmbH, Freiburg, Germany) and antiSmD titres (Hycor Biomedical, Indianapolis, IN, USA), according to the provider's instructions.

\section{Statistical analysis}

Statistical analysis was performed using the GraphPad Prism version 5.0 (GraphPad Software Inc, San Diego, CA, USA; http://www.graphpad.com) and spss version $13 \cdot 0$ (SPSS Inc., Chicago, IL, USA) software. The differences between the groups were evaluated with the MannWhitney $U$-test. Correlations between the variables were expressed using the non-parametric Spearman's correlation coefficients. The categorical variables were compared with the Fisher's exact test. All the tests were two-tailed.

\section{Results}

Differences in the serum concentration of anti-EBNA-1 IgG-type antibodies comparing patients with MS, SLE and healthy subjects

Compared to the healthy controls, the levels of IgG-type anti-EBNA-1 antibody were significantly higher in the sera of both the MS and the SLE patients (Fig. 1). Comparing the two patient groups revealed a higher anti-EBNA-1 antibody concentration in the MS than in the SLE group (Fig. 1). Seronegative $(<20 \mathrm{U} / \mathrm{ml})$ samples occurred significantly less frequently among both the MS patients (five of

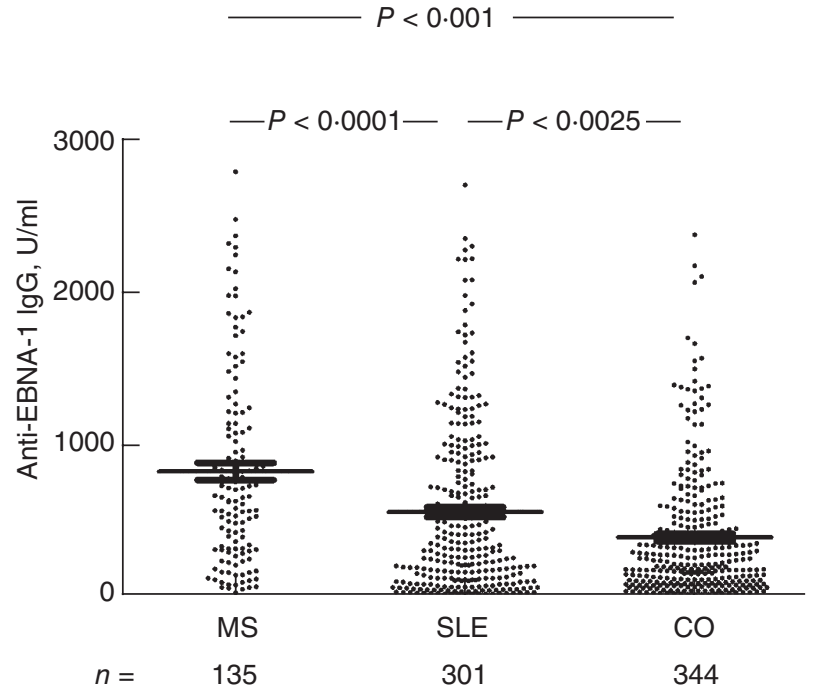

Fig. 1. The levels of immunoglobulin (Ig)G-type antibodies to whole Epstein-Barr nuclear antigen 1 (EBNA-1), in the sera of EBNA-1-positive patients with multiple sclerosis (MS) or systemic lupus erythematosus (SLE) and in healthy subjects. EBNA-1 positivity (> $20 \mathrm{U} / \mathrm{ml}$ ) was found in 96\% (130 of 135) of MS patients, in 95\% (286 of 301) of SLE patients and in 90\% (310 of 345) of healthy control subjects. $P$-values of non-parametric analysis of variance (Kruskal-Wallis) test followed by Dunn's post-hoc test are shown.

135) $(P=0 \cdot 0261)$ and SLE patients $(15$ of 301$)(P<0 \cdot 0258$, Fisher's exact test) than among the control samples (35 of 345). When the EBV-negative subjects were excluded from the comparative analysis, significant differences were still found between the IgG EBNA-1 response levels of EBVpositive MS patients [654.8 (313.8-1138.0) AU/ml], SLE patients [344.4 (120.2-890.7) AU/ml] and healthy controls [202.0 (87.2-453.9) AU/ml]. Both high (>median, $300 \mathrm{U} /$ $\mathrm{ml}$ ) and very high (in the highest quartile $(>720 \mathrm{U} / \mathrm{ml}$ ) values were found significantly more frequently in both the MS and SLE patients' sera compared to healthy controls (Fig. 2). When comparing the subgroups of MS patients, no difference was found between MS patients without treatment $[578.0(246 \cdot 0-1060 \cdot 0) \mathrm{AU} / \mathrm{ml}]$ or with treatment [641.9 (290.0-1591.0) AU/ml] $(P=0.2581)$. Similarly, no difference was found between SLE patients without treatment [301.7 (71.1-949.4) AU/ml] or with treatment [343.9 $(103 \cdot 5-712 \cdot 3) \mathrm{AU} / \mathrm{ml}](P=0 \cdot 9099)$.

The frequency of HLA-DRB $1^{\star} 15: 01$ carriers among MS or SLE patients and healthy subjects

The frequency of this allele was determined in representative groups of 268 SLE patients and 90 MS patients, as well as 282 healthy controls (no DNA samples were available for the remaining patients) (Fig. 3 ). Carriers of the HLA$\mathrm{DRB}^{\star} 15: 01$ allele were significantly more common among the MS patients than in the group of healthy controls. When the subjects were divided according to sex, the 
Fig. 2. Frequency of the (a) 'high' (> $300 \mathrm{U} / \mathrm{ml}$, median of all values) and (b) 'very high' (> $720 \mathrm{U} / \mathrm{ml}$, in the highest quartile of all values) anti-Epstein-Barr nuclear antigen 1 (EBNA-1)titres in the sera of multiple sclerosis (MS) and systemic lupus erythematosus (SLE) patients, as well as healthy controls (CO). Both 'high' and 'very high' titres were found significantly more frequently in both the MS and SLE patients' sera compared to the healthy controls, as shown by the percentages.

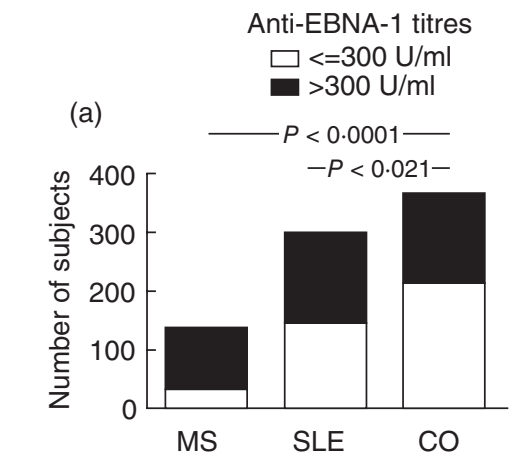

$\%$ high titres 74 frequency of the HLA-DRB1*15:01 carrier state was significantly higher among MS patients than among healthy subjects, both in females and in males $(P=0.0084$ and $P=0 \cdot 0058)$. This frequency was also significantly higher in SLE patients than in healthy controls, although the difference was smaller (Fig. 3).

The relationship between the carrier state of the HLA-DRB1 ${ }^{\star}$ 15:01 allele and anti-EBNA-1 antibody levels in patients with MS or SLE and in healthy controls

Next, we compared the levels of anti-EBNA-1 antibodies separately in DRB1*15:01 carriers and non-carriers in patients and controls. Both in carriers and non-carriers, compared to the healthy controls, the serum concentration of IgG antibodies to EBNA-1 was significantly higher both in MS and in SLE patients, although the difference was greater in the case of the former (Table 1).

Next we investigated if the effect of the HLA-DRB1 ${ }^{\star} 15: 01$ carrier state and the high titre of the anti-EBNA-1 antibodies are additive. When the distribution of subjects with neither, and both of these factors, was calculated, we found highly significant $(P=0 \cdot 0008)$ differences between controls subjects and SLE patients: 152 of 282 (54\%) and 15 of 282 (5\%) of the controls had none of the risk factors and both

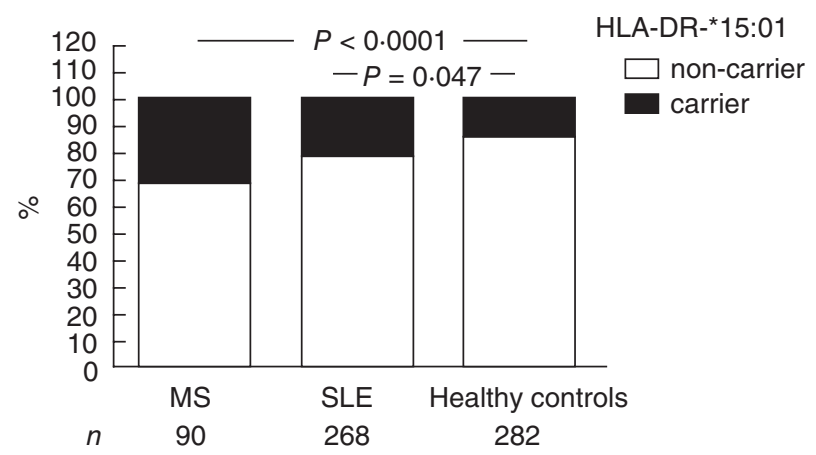

Fig. 3. The distribution of human leucocyte antigen (HLA)-DRB1 ${ }^{\star} 15: 01$ carriers within the study groups. $P$-values for Fisher's exact test are indicated.

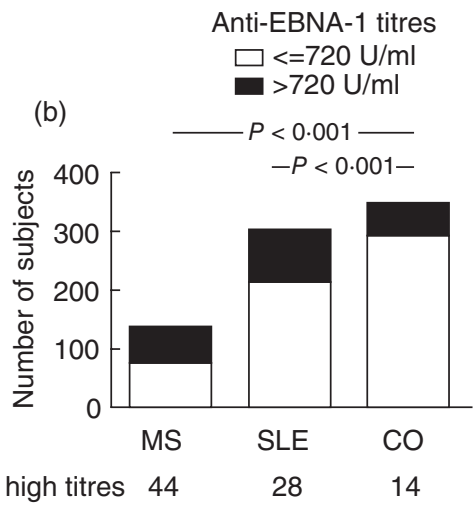

risk factors, respectively, while for the SLE patients the same proportions were 107 of 268 (40\%) and 32 of 268 (12\%), respectively. In the case of MS, even higher differences $(P<0.0001)$ were observed: no risk factor, 21 of $90(23 \%)$; both risk factors, 27 of $90(30 \%)$.

Differences in the serum concentration of the antibodies to the aa35-58 and aa398-404 fragments of the EBNA-1 in patients with MS or SLE, and in healthy subjects

Antibodies against the aa35-58 epitope region of EBNA-1 presented significantly elevated levels both in MS patients [26.6 (2.9-103.0) AU/ml; $P=0.0037]$ and in SLE patients [33.2 (3.3-129.5) AU/ml; $P<0 \cdot 0001]$, compared to healthy controls [7.5 (0-32.6) AU/ml] (Fig. 4a). We could not find a difference in the study group regarding the frequency of patients with undetectable antibodies against the aa35-58 fragment of the EBNA-1. As the N-terminal 35-58 sequence of EBNA-1 is highly homologous to the C-terminal 95-119 region of the ribonucleoprotein $\mathrm{SmD}$, we also determined

Table 1. The levels of immunoglobulin (Ig)G-type antibodies to Epstein-Barr nuclear antigen 1 (EBNA-1)in the sera of patients with multiple sclerosis (MS) or with systemic lupus erythematosus (SLE) and in healthy subjects, who carry or do not carry the human leucocyte antigen (HLA)-DRB1*15:01.

\begin{tabular}{lrl}
\hline Group & $\begin{array}{c}\text { Number } \\
\text { of subjects }\end{array}$ & \multicolumn{1}{c}{$\begin{array}{c}\text { Anti-EBNA-1 IgG, U/ml } \\
\text { median (interquartile range) }\end{array}$} \\
\hline $\begin{array}{l}\text { HLA-DRB1 }{ }^{*} 15: 01 \text { carriers } \\
\text { MS patients }\end{array}$ & 31 & $596 \cdot 0(338 \cdot 8-1100 \cdot 8)^{* * *}+++$ \\
SLE patients & 53 & $386 \cdot 9(102 \cdot 7-963 \cdot 1)^{*}$ \\
Healthy subjects & 42 & $198 \cdot 7(71 \cdot 7-427 \cdot 0)$ \\
HLA-DRB1 $15: 01$ non-carriers & \\
MS patients & 59 & $438 \cdot 0(146 \cdot 0-825 \cdot 0)^{* *+++}$ \\
SLE patients & 215 & $304 \cdot 7(103 \cdot 1-726 \cdot 1)^{* * *}$ \\
Healthy subjects & 240 & $183 \cdot 7(76 \cdot 3-455 \cdot 8)$ \\
\hline
\end{tabular}

$+++P<0.001$ between the two patient groups. ${ }^{* *} P<0 \cdot 001$ ${ }^{*} P<0 \cdot 01 ;{ }^{\star} P<0.05$ compared to the healthy control group. Nonparametric analysis of variance (Kruskal-Wallis) test followed by Dunn's post-hoc test. 
Fig. 4. The levels of immune globulin (Ig)G-type antibodies to the (a) aa35-58 or to the (b) aa398-404 fragments of Epstein-Barr nuclear antigen 1 (EBNA-1) molecule, in the sera of patients with multiple sclerosis (MS) or systemic lupus erythematosus (SLE) and in healthy subjects. $P$-values of non-parametric analysis of variance (Kruskal-Wallis) test followed by Dunn's post-hoc test are shown. n.s.: not significant. (a)

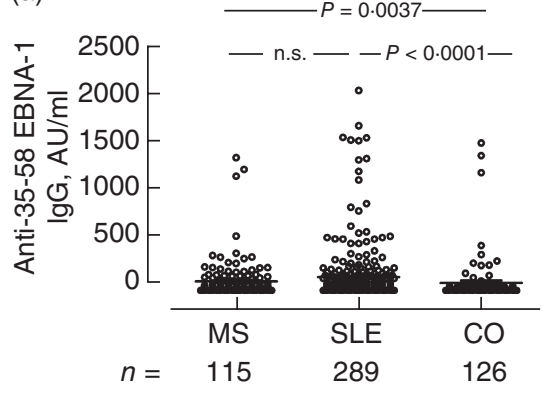

(b)

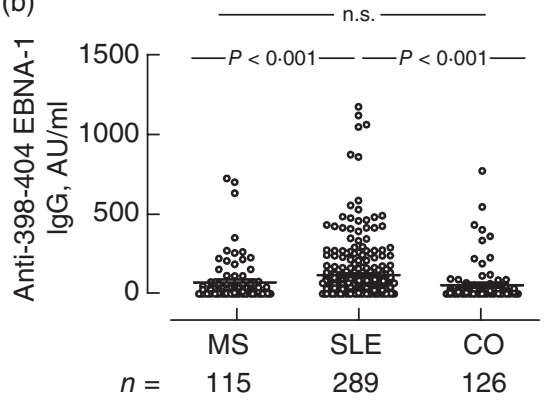

the anti-SmD titres in SLE patients. A significant correlation was found between the anti-35-58 EBNA-1 levels and the anti-SmD titres $(r=0 \cdot 2474, P<0 \cdot 0001)$, as well as a moderate but significant correlation with the anti-SmB/B' titres $(r=0 \cdot 1402, P=0 \cdot 0258)$.

Compared to healthy controls $[7 \cdot 3(0 \cdot 0-31 \cdot 1) \mathrm{AU} / \mathrm{ml}]$, the serum concentration of antibodies against the aa398404 epitope of the EBNA-1 was, significantly, sixfold higher in the sera of the SLE patients [44.8 (12.7-127.6) AU/ml]. By contrast, no significant difference could be found between the MS patients $[10 \cdot 3(0.5-41.6) \mathrm{AU} / \mathrm{ml}]$ and healthy controls (Fig. 4b). The same reactivity patterns were ascertained when the results were stratified according to the HLA-DRB1 ${ }^{\star}$ 15:01 carrier state (data not shown). Antibodies against the aa398-404 epitope of the EBNA-1 were undetectable in 28 of 115 MS patients $(P=0.0091$ compared to the controls, $P<0.0001$ compared to the SLE patients, Fisher's exact test), in 22 of 289 SLE patients $(P<0.0001)$ and in 51 of 126 control samples.

We found a significant correlation between the titres of antibodies against the aa398-404 epitope of the EBNA-1 and the anti-SmB/B' titres $(r=0.3286, P<0.0001)$, and a slight but still significant correlation with the anti-SmD titres $(r=0 \cdot 1517, P=0 \cdot 0095)$.

\section{Discussion}

Our paper reports two novel findings. First, we extended to SLE patients the previous observations of several authors, indicating that the HLA-DRB1*15:01 carrier state and the high serum concentration of IgG-type anti-EBNA-1 antibodies are independent and additive risk factors of MS. We have demonstrated that, compared to healthy controls, the level of anti-EBNA-1 IgG is higher in SLE patients, whether or not they carry the HLA-DRB1*15:01 allele, and the effect of the two risk factors are additive. Secondly, we found that the serum concentration of IgG-type antibodies against a particular EBNA-1 epitope (aa398-404, PPPGRRP), which has been found previously to cross-react with an autoantibody (anti-SmB/B') characteristic for SLE, is elevated in SLE but not in MS. Interestingly, titres of antibodies against the aa35-58 EBNA-1 epitope region, which is highly homologous to the C-terminal 95-119 region of $\mathrm{SmD}$, were elevated both in MS and SLE patients.

Sundstrom et al. [29] were the first to demonstrate by multivariate logistic regression analysis that EBNA-1 reactivity and HLA-DRB1 ${ }^{\star}$ 15:01 are statistically independent risk factors for MS. Later, the same group [12] found that the levels of antibodies against several epitopes of EBNA-1 are also significantly higher in MS patients than in healthy controls, regardless of whether or not they carry the HLA$\mathrm{DRB}^{\star}{ }^{\star}$ 15:01 antigen. In accordance with these findings, Waubant et al. [30] found that EBNA-1 seropositivity was associated with an almost fourfold risk of MS in patients with early paediatric MS. Interestingly, in a further study children with MS did not differ from age-matched healthy controls in seroprevalence of further childhood viruses [31].

Furthermore, another group [11] showed that the association between anti-EBNA-1 titres and MS risk was not affected by adjustment for DR15, and was similar in DR15positive and DR15-negative women. More recently [13], the same group reported on the combined effects of smoking, anti-EBNA antibodies and HLA-DRB1*15:01 on MS risk. Sundqvist et al. [32] studied the interactions of three MS risk factors in association with high anti-EBNA-1 titres (and those of the anti-385-420 fragment of the EBNA-1, HLA$\mathrm{DRB}^{\star}{ }^{\star 15: 01)}$ as well as in the absence of HLA-A ${ }^{\star} 02$. They found a strong interaction among these risk factors and concluded that the mechanism through which HLA genes influence the risk of MS may be related to the immune control of EBV infection. Our present findings are in complete accord with these results. Compared to healthy control subjects, the serum concentration of anti-EBNA-1 antibodies was significantly $(P<0.001)$ higher in the sera of both HLA-DRB1 ${ }^{\star}$ 15:01 carrier and non-carrier MS patients. By contrast, our present findings are at variance with the recent data from Irizar et al. [33], as in the Hungarian population we did not find the association between HLA-DRB1*15:01 and MS to be female-specific.

Only speculative explanations have been published to date for the presumed causes of the elevated anti-EBNA-1 IgG antibody levels in MS. According to Levin et al. [7], the elevation of antibody titres to EBNA-1, seen in future MS 
patients during their early 20s, suggests a more severe or more recent primary infection or reactivation of a preexisting infection, accompanied by a vigorous cellular immune response. Very recently, Santon et al. [34] detected dual infection by both types ( 1 and 2 ) of EBV in 63 patients (90\%) and 46 controls (37.4\%). Logistic regression models showed a significant $(P<0.001)$ association between MS and dual-type infection. It can be assumed that this dual infection may result in the elevation of anti-EBNA-1 IgG titres.

Considering that SLE also seems to be associated with EBV infection $[14,15,35,36]$, and HLA-DRB1 ${ }^{\star} 15: 01$ is one of the genetic risk factors for SLE [19,20], we performed a similar study of SLE patients. To the best of our knowledge, until now the interaction between high-titre anti-EBNA-1 antibodies and the carrier state of HLA-DRB1*15:01 has not been studied. In accordance with earlier work $[14,15]$, but in contrast to a recent study [16], we found that the titre of the anti-EBNA-1 antibody is significantly higher in SLE patients than in healthy controls. Performing this comparison in carriers and non-carriers of HLA-DRB $1^{\star} 15: 01$, we found the same difference for both groups, indicating that high EBNA-1 reactivity and the carrier state of HLA $\mathrm{DRB}^{\star}{ }^{\star} 15: 01$ are statistically independent and additive risk factors for both MS and SLE.

In the second part of the study, we found substantial differences between MS and SLE patients regarding the level of IgG-type antibodies to an EBNA-1 epitope (aa398-404, PPPGRRP). The serum concentration of this type of antibody was significantly higher in SLE patients than in healthy controls, while no difference could be found between controls and MS patients. The high serum concentration of anti-aa398-404 EBNA-1 antibodies is not surprising in SLE, as many observations in patients [14] and data from animal experiments [37] demonstrated a strong cross-reaction between this EBNA-1 epitope and an epitope $\left(B^{\prime} / B\right)$ of the Sm autoantigen, and autoantibodies against the latter are characteristic in SLE. However, it was demonstrated recently [12] that, in MS, the elevated level of anti-EBNA-1 includes antibodies directed against several epitopes of the protein, but anti-PPPGRRP antibodies have not been studied previously in MS patients. Nevertheless, Sundstrom et al. [12] measured antibodies to a larger EBNA-1 fragment (391-410, SGSPPRRPPPGRRPFFHPVG), which includes the 398-404 fragment, and found only a small difference $(P=0 \cdot 047)$ in the antibody levels of MS patients and controls. Although antibodies against the aa35-58 epitope region of EBNA-1 showed a significantly higher level in SLE or MS patients, no difference was found among the study groups regarding the frequency of patients with undetectable antibodies against the aa35-58 epitope region of the EBNA-1. Our results are in accordance with the findings of Marchini et al. [25], who described that antibodies to the aa35-58 EBNA-1 fragment are produced in controls and in EBV-related diseases, but only SLE sera contain anti-viral antibodies cross-reactive with the SmD autoantigen. Thus, our present findings suggest that the elevated level of the anti-PPPGRRP antibody is characteristic of and specific for SLE.

Earlier, we summarized [4] findings indicating differences in the relationship of EBV with the two autoimmune diseases, MS and SLE. The incidence of MS [38], but not of SLE [39], is markedly higher in those who had had infectious mononucleosis previously. While the viral load is extremely high in SLE [40,41], in MS it is similar to that seen in the healthy population [42]. Our present finding on anti-PPPGRRP antibody levels also supports this conclusion. Thus, our present observations are in line with the literature data, and indicate that there are both similarities and differences in the relationship of EBV with two autoimmune diseases, MS and SLE. Studies into these connections may provide new data on the nature of the associations between EBV infection and these autoimmune disorders.

\section{Acknowledgements}

The Varelisa ${ }^{\circledR}$ Sm Antibodies assay for the determination of anti-SmB/B' titres was a generous gift from Dr Wolfgang Papisch on behalf of Thermo Fisher Scientific, Phadia GmbH (Freiburg, Germany).

\section{Disclosure}

Our work was supported by grant no. 80842 of the National Research Fund to G.F. and by grant no. OTKA-NKTH 100687 to Z.P.

\section{References}

1 Csuka D, Banati M, Rozsa C, Fust G, Illés Z. High anti-EBNA-1 IgG levels are associated with early-onset myasthenia gravis. Eur J Neurol 2012; 19:842-6.

2 Niller HH, Wolf H, Minarovits J. Regulation and dysregulation of Epstein-Barr virus latency: implications for the development of autoimmune diseases. Autoimmunity 2008; 41:298-328.

3 Ascherio A. Epstein-Barr virus in the development of multiple sclerosis. Exp Rev Neurother 2008; 8:331-3.

4 Fust G. The role of the Epstein-Barr virus in the pathogenesis of some autoimmune disorders - similarities and differences. Eur J Microbiol Immunol 2011; 1:267-78.

5 Ascherio A, Munger KL, Lennette ET et al. Epstein-Barr virus antibodies and risk of multiple sclerosis: a prospective study. JAMA 2001; 286:3083-8.

6 Sundstrom P, Juto P, Wadell G et al. An altered immune response to Epstein-Barr virus in multiple sclerosis: a prospective study. Neurology 2004; 62:2277-82.

7 Levin LI, Munger KL, Rubertone MV et al. Temporal relationship between elevation of Epstein-Barr virus antibody titers and initial onset of neurological symptoms in multiple sclerosis. JAMA 2005; 293:2496-500.

8 DeLorenze GN, Munger KL, Lennette ET, Orentreich N, Vogelman JH, Ascherio A. Epstein-Barr virus and multiple sclerosis: evidence of association from a prospective study with long-term follow-up. Arch Neurol 2006; 63:839-44. 
9 Levin LI, Munger KL, O'Reilly EJ, Falk KI, Ascherio A. Primary infection with the Epstein-Barr virus and risk of multiple sclerosis. Ann Neurol 2010; 67:824-30.

10 Munger K, Levin L, O’Reilly E, Falk K, Ascherio A. Anti-EpsteinBarr virus antibodies as serological markers of multiple sclerosis: a prospective study among United States military personnel. Mult Scler 2011; 17:1185-93.

11 De Jager PL, Simon KC, Munger KL, Rioux JD, Hafler DA, Ascherio A. Integrating risk factors: HLA-DRB1 ${ }^{\star} 1501$ and Epstein-Barr virus in multiple sclerosis. Neurology 2008; 70:1113-8.

12 Sundstrom P, Nystrom M, Ruuth K, Lundgren E. Antibodies to specific EBNA-1 domains and HLA DRB1*1501 interact as risk factors for multiple sclerosis. J Neuroimmunol 2009; 215:102-7.

13 Simon KC, van der Mei IA, Munger KL et al. Combined effects of smoking, anti-EBNA antibodies, and HLA-DRB1*1501 on multiple sclerosis risk. Neurology 2010; 74:1365-71.

14 McClain MT, Rapp EC, Harley JB, James JA. Infectious mononucleosis patients temporarily recognize a unique, cross-reactive epitope of Epstein-Barr virus nuclear antigen-1. J Med Virol 2003; 70:253-7.

15 Toussirot E, Roudier J. Epstein-Barr virus in autoimmune diseases. Best Pract Res Clin Rheumatol 2008; 22:883-96.

16 Esen BA, Yilmaz G, Uzun S et al. Serologic response to EpsteinBarr virus antigens in patients with systemic lupus erythematosus: a controlled study. Rheumatol Int 2012; 32:79-83.

17 Zandman-Goddard G, Berkun Y, Barzilai O et al. Exposure to Epstein-Barr virus infection is associated with mild systemic lupus erythematosus disease. Ann NY Acad Sci 2009; 1173: 658-63.

18 Truedsson L, Sturfelt G, Johansen P, Nived O, Thuresson B. Sharing of MHC haplotypes among patients with systemic lupus erythematosus from unrelated Caucasian multicase families: disease association with the extended haplotype (HLA-B8, SC01, DR17)]. J Rheumatol 1995; 22:1852-61.

19 Podrebarac TA, Boisert DM, Goldstein R. Clinical correlates, serum autoantibodies and the role of the major histocompatibility complex in French Canadian and non-French Canadian Caucasians with SLE. Lupus 1998; 7:183-91.

20 Marchini M, Antonioli R, Lleo A et al. HLA class II antigens associated with lupus nephritis in Italian SLE patients. Hum Immunol 2003; 64:462-8.

21 Sundar K, Jacques S, Gottlieb P et al. Expression of the EpsteinBarr virus nuclear antigen-1 (EBNA-1) in the mouse can elicit the production of anti-dsDNA and anti-Sm antibodies. J Autoimmun 2004; 23:127-40.

22 McClain MT, Poole BD, Bruner BF, Kaufman KM, Harley JB, James JA. An altered immune response to Epstein-Barr nuclear antigen 1 in pediatric systemic lupus erythematosus. Arthritis Rheum 2006; 54:360-8.

23 Harley JB, James JA. Epstein-Barr virus infection induces lupus autoimmunity. Bull NYU Hosp Jt Dis 2006; 64:45-50.

24 Poole BD, Templeton AK, Guthridge JM, Brown EJ, Harley JB, James JA. Aberrant Epstein-Barr viral infection in systemic lupus erythematosus. Autoimmun Rev 2009; 8:337-42.

25 Marchini B, Dolcher MP, Sabbatini A, Klein G, Migliorini P. Immune response to different sequences of the EBNA I molecule in Epstein-Barr virus-related disorders and in autoimmune diseases. J Autoimmun 1994; 7:179-91.

26 Goris A, Walton A, Ban M, Dubois B, Compston A, Sawcer S. A Taqman assay for high-throughput genotyping of the multiple sclerosis-associated HLA-DRB1*1501 allele. Tissue Antigens 2008; 72:401-3.

27 Tam JP. Synthetic peptide vaccine design: synthesis and properties of a high-density multiple antigenic peptide system. Proc Natl Acad Sci USA 1988; 85:5409-13.

28 Bartos A, Uray K, Hudecz F. New biotin derivatives for labeling and solubilizing IgG peptides. Biopolymers 2009; 92:110-5.

29 Sundstrom P, Nystrom L, Jidell E, Hallmans G. EBNA-1 reactivity and HLA DRB1*1501 as statistically independent risk factors for multiple sclerosis: a case-control study. Mult Scler 2008; 14:1120-2.

30 Waubant E, Mowry EM, Krupp L et al. Common viruses associated with lower pediatric multiple sclerosis risk. Neurology 2011; 76:1989-95.

31 Banwell B, Krupp L, Kennedy J et al. Clinical features and viral serologies in children with multiple sclerosis: a multinational observational study. Lancet Neurol 2007; 6:773-81.

32 Sundqvist E, Sundstrom P, Linden M et al. Epstein-Barr virus and multiple sclerosis: interaction with HLA. Genes Immun 2012; 13:14-20.

33 Irizar H, Munoz-Culla M, Zuriarran O et al. HLA-DRB1*15:01 and multiple sclerosis: a female association? Mult Scler 2012; 18:569-77.

34 Santon A, Cristobal E, Aparicio M, Royuela A, Villar LM, AlvarezCermeno JC. High frequency of co-infection by Epstein-Barr virus types 1 and 2 in patients with multiple sclerosis. Mult Scler 2011; 17:1295-300.

35 James JA, Neas BR, Moser KL et al. Systemic lupus erythematosus in adults is associated with previous Epstein-Barr virus exposure. Arthritis Rheum 2001; 44:1122-6.

36 James JA, Kaufman KM, Farris AD, Taylor-Albert E, Lehman TJ, Harley JB. An increased prevalence of Epstein-Barr virus infection in young patients suggests a possible etiology for systemic lupus erythematosus. J Clin Invest 1997; 100:3019-26.

37 Poole BD, Gross T, Maier S, Harley JB, James JA. Lupus-like autoantibody development in rabbits and mice after immunization with EBNA-1 fragments. J Autoimmun 2008; 31:362-71.

38 Thacker EL, Mirzaei F, Ascherio A. Infectious mononucleosis and risk for multiple sclerosis: a meta-analysis. Ann Neurol 2006; 59:499-503.

39 Ulff-Moller CJ, Nielsen NM, Rostgaard K, Hjalgrim H, Frisch M. Epstein-Barr virus-associated infectious mononucleosis and risk of systemic lupus erythematosus. Rheumatology (Oxf) 2010; 49:1706-12.

40 Moon UY, Park SJ, Oh ST et al. Patients with systemic lupus erythematosus have abnormally elevated Epstein-Barr virus load in blood. Arthritis Res Ther 2004; 6:R295-302.

41 Kang I, Quan T, Nolasco H et al. Defective control of latent Epstein-Barr virus infection in systemic lupus erythematosus. J Immunol 2004; 172:1287-94.

42 Lindsey JW, Hatfield LM, Crawford MP, Patel S. Quantitative PCR for Epstein-Barr virus DNA and RNA in multiple sclerosis. Mult Scler 2009; 15:153-8. 